\title{
TIME DECAY FOR THE NONLINEAR BEAM EQUATION*
}

\author{
STEVEN P. LEVANDOSKY ${ }^{\dagger}$ AND WALTER A. STRAUSS ${ }^{\ddagger}$
}

\begin{abstract}
We derive an analogue of Morawetz' radial identity for the case of a fourth-order wave equation. It follows that, for a nonlinear term with repelling sign, all the solutions decay to zero in a certain sense as $t \rightarrow \infty$. This is an initial step in the construction of a scattering theory for such nonlinear waves.
\end{abstract}

Cathleen Morawetz wrote a short but seminal paper in 1968 on the nonlinear Klein-Gordon equation in which she proved her important Radial Identity and deduced the decay of the local energy of solutions. This identity subsequently had several other important consequences. It was the key ingredient in the proof that all states are scattering states for the Klein-Gordon equation with nonlinear terms with repelling signs, proved in several stages of increasing generality $[\mathrm{MS}][\mathrm{Br}]$. Analogues of it were also used in the scattering theory for nonlinear Schrödinger equations [LS][GV1], for nonlinear wave equations with zero mass [GV2], and for Hartree equations [GV3]. It was also recently used to prove the well-posedness of critical nonlinear Schrödinger equations $[\mathrm{B}][\mathrm{G}]$. A pseudodifferential generalization of it was also used to prove that the local energy of a solution of the linear wave equation satisfying the Dirichlet boundary condition in the exterior of a non-trapping obstacle decays to zero [MRS].

However, nothing of this sort is known for an equation of order four like

$$
u_{t t}+\Delta^{2} u+f(u)=0
$$

the simplest nonlinear perturbation of the classical vibrating beam equation. A general theory of such a nonlinear beam equation was considered in two papers by the first author, including the following results. (i) The local well-posedness in $H^{2}\left(\mathbb{R}^{n}\right) \times$ $L^{2}\left(\mathbb{R}^{n}\right)$ for $f(u)=u+O\left(|u|^{p}\right)$ with $1<p<1+\frac{8}{n-4}$. (ii) Low energy scattering in $H^{2}\left(\mathbb{R}^{n}\right) \times L^{2}\left(\mathbb{R}^{n}\right)$ for $f(u)=u+|u|^{p-1} u$ with $p \geq 1+\frac{8}{n}$. (iii) Stability and instability of solitary and standing waves for the case of a nonlinear term of attractive sign like $f(u)=u-|u|^{p-1} u$ with $1<p<1+\frac{8}{n-4}$.

In this note we consider the global scattering problem for the fourth-order nonlinear beam equation. By a rather long but totally explicit calculation, we prove an exact analogue of Morawetz' Radial Identity. The integrability in time of certain quantities follows automatically. We conjecture that, for the nonlinear beam equation for $f(u)=u+|u|^{p-1} u$ with $1+\frac{8}{n}<p<1+\frac{8}{n-4}$, all states are scattering states. This note is a report of work in progress and at the end of it we discuss some supporting evidence for the scattering conjecture.

1. The identity. For equation (1) the energy

$$
E=\int\left\{\frac{1}{2} u_{t}^{2}+\frac{1}{2}|\Delta u|^{2}+F(u)\right\} d x
$$

* Received March 1, 2000.

$\dagger$ Department of Mathematics, Stanford University, Stanford, CA 94305, U.S.A. (spl@math. stanford.edu).

$\ddagger$ Department of Mathematics, Lefschetz Center for Dynamical Systems, Brown University, Providence, RI 02912, U.S.A. (wstrauss@math.brown.edu). Supported in part by NSF grant 9703695. 
is a constant, where $\int \ldots d x$ means the integral over all of $\mathbb{R}^{n}$ and $F^{\prime}=f, F(0)=0$.

THEOREM 1. Let $n \geq 5$. Assume that $u$ is a solution of (1) smooth enough, and small enough at infinity, to justify certain integrations by parts. Then

$$
\begin{aligned}
0 & =\frac{d}{d t} \int u_{t}\left(u_{r}+\frac{n-1}{2 r} u\right) d x \\
& +\frac{1}{2}(n-1)(n-3) \int \frac{1}{r^{3}} u_{r}^{2} d x+\frac{1}{2}\left(n^{2}+2 n-19\right) \int \frac{1}{r^{3}}\left(|\nabla u|^{2}-u_{r}^{2}\right) d x+P \\
& +\frac{3}{4}(n-1)(n-3)(n-5) \int \frac{1}{r^{5}} u^{2} d x+\frac{1}{2}(n-1) \int \frac{1}{r}[u f(u)-2 F(u)] d x
\end{aligned}
$$

where $P \geq 0$ will be given explicitly below, $r=|x|$ and $u_{r}=\frac{x}{r} \cdot \nabla u$.

Proof. We multiply equation (1) by the skew-adjoint expression $B u=u_{r}+\frac{n-1}{2 r} u$. Then the first and third terms in (1) give

$$
\left\{\partial_{t}^{2} u+f(u)\right\} B u=\partial_{t}\left\{\partial_{t} u B u\right\}-\frac{x_{k}}{r} \partial_{t} u\left\{\partial_{k} \partial_{t} u+\partial_{k} F(u)\right\}+\frac{n-1}{2 r}\left\{-\left(\partial_{t} u\right)^{2}+u f(u)\right\}
$$

where $\partial_{k}=\partial / \partial x_{k}$ and we sum over repeated indices $k$. We shall also use the symbol $\approx$ to indicate that two quantities differ by pure spatial derivatives. Then we can write

$$
\begin{aligned}
\left\{\partial_{t}^{2} u+f(u)\right\} B u & \approx \partial_{t}\left\{\partial_{t} u B u\right\}+\partial_{k}\left(\frac{x_{k}}{r}\right)\left\{\frac{1}{2}\left(\partial_{t} u\right)^{2}-F(u)\right\}+\frac{n-1}{2 r}\left\{-\left(\partial_{t} u\right)^{2}+u f(u)\right\} \\
& =\partial_{t}\left\{\partial_{t} u B u\right\}+\frac{n-1}{2 r}\{u f(u)-2 F(u)\} .
\end{aligned}
$$

Thus we have accounted for the first and last terms in (2). It remains to calculate $\left(\Delta^{2} u\right)(B u)$.

We begin by using the relation

$$
u_{i i j j} u_{k}=\left(u_{i j j} u_{k}-u_{j j} u_{i k}\right)_{i}+\left(\frac{1}{2}\left(u_{i i}\right)^{2}\right)_{k}
$$

where the subscripts denote partial derivatives, to compute

$$
\begin{aligned}
\left(\Delta^{2} u\right) u_{r} & =u_{i i j j} u_{k} \frac{x_{k}}{r}=\frac{x_{k}}{r}\left(u_{i j j} u_{k}-u_{j j} u_{i k}\right)_{i}+\frac{x_{k}}{2 r}\left(\left(u_{i i}\right)^{2}\right)_{k} \\
& \approx-\left(\frac{x_{k}}{r}\right)_{i}\left(u_{i j j} u_{k}-u_{j j} u_{i k}\right)-\frac{x_{k}}{2 r}\left(u_{i i}\right)^{2} \\
& =-\left(\frac{\delta_{i k}}{r}-\frac{x_{i} x_{k}}{r^{3}}\right)\left(u_{i j j} u_{k}-u_{j j} u_{i k}\right)-\frac{n-1}{2 r}\left(u_{i i}\right)^{2} \\
& =\frac{1}{r}\left(u_{i i} u_{j j}-u_{i j j} u_{i}\right)-\frac{n-1}{2 r}\left(u_{i i}\right)^{2}+\frac{x_{i} x_{k}}{r^{3}}\left(u_{i j j} u_{k}-u_{j j} u_{i k}\right) .
\end{aligned}
$$

and

$$
\begin{aligned}
\left(\Delta^{2} u\right)\left(\frac{n-1}{2 r} u\right) & =\frac{n-1}{2 r} u_{i i j j} u=\frac{n-1}{2 r}\left(\left(u_{i j j} u-u_{j j} u_{i}\right)_{i}+u_{j j} u_{i i}\right) \\
& \approx-\frac{n-1}{2}\left(\frac{1}{r}\right)_{i}\left(u_{i j j} u-u_{j j} u_{i}\right)+\frac{n-1}{2 r}\left(u_{i i}\right)^{2} \\
& =\frac{n-1}{2} \frac{x_{i}}{r^{3}}\left(u_{i j j} u-u_{j j} u_{i}\right)+\frac{n-1}{2 r}\left(u_{i i}\right)^{2}
\end{aligned}
$$


Thus

$$
\begin{aligned}
\left(\Delta^{2} u\right)(B u) & =\left(\Delta^{2} u\right)\left(u_{r}+\frac{n-1}{2 r} u\right) \\
& \approx \frac{1}{r}\left(u_{i i} u_{j j}-u_{i j j} u_{i}\right)+\frac{x_{i} x_{k}}{r^{3}}\left(u_{i j j} u_{k}-u_{j j} u_{i k}\right)+\frac{n-1}{2} \frac{x_{i}}{r^{3}}\left(u_{i j j} u-u_{j j} u_{i}\right) \\
& \equiv I+I I+I I I .
\end{aligned}
$$

The first term $I$ we rewrite as

$$
\begin{aligned}
I & \approx u_{i j}\left(\frac{1}{r} u_{i}\right)_{j}-u_{i}\left(\frac{1}{r} u_{j j}\right)_{i} \\
& \approx \frac{2}{r} u_{i j}^{2}+2 u_{i j} u_{i}\left(\frac{1}{r}\right)_{j}-u_{j j} u_{i}\left(\frac{1}{r}\right)_{i} .
\end{aligned}
$$

The second term II becomes

$$
\begin{aligned}
I I & \approx\left(u_{j} u_{i j k}-u_{i j} u_{j k}\right)\left(\frac{x_{i} x_{k}}{r^{3}}\right)+\left(u_{j} u_{i k}-u_{k} u_{i j}\right)\left(\frac{x_{i} x_{k}}{r^{3}}\right)_{j} \\
& \approx-2 u_{i j} u_{j k}\left(\frac{x_{i} x_{k}}{r^{3}}\right)+\left(u_{j} u_{i k}-u_{k} u_{i j}\right)\left(\frac{x_{i} x_{k}}{r^{3}}\right)_{j}-u_{i j} u_{j}\left(\frac{x_{i} x_{k}}{r^{3}}\right)_{k}
\end{aligned}
$$

and the third term $I I I$ can be written as

$$
I I I=-\frac{n-1}{2}\left(\frac{1}{r}\right)_{i}\left(u_{i j j} u-u_{j j} u_{i}\right) .
$$

Adding $I, I I$ and $I I I$, we obtain

$$
\begin{aligned}
\left(\Delta^{2} u\right)(B u) & \approx \frac{2}{r} u_{i j}^{2}-2 u_{i j} u_{j k}\left(\frac{x_{i} x_{k}}{r^{3}}\right)+2 u_{i j} u_{i}\left(\frac{1}{r}\right)_{j} \\
& -u_{j j} u_{i}\left(\frac{1}{r}\right)_{i}+\left(u_{j} u_{i k}-u_{k} u_{i j}\right)\left(\frac{x_{i} x_{k}}{r^{3}}\right)_{j} \\
& -u_{i j} u_{j}\left(\frac{x_{i} x_{k}}{r^{3}}\right)_{k}-\frac{n-1}{2}\left(\frac{1}{r}\right)_{i}\left(u_{i j j} u-u_{j j} u_{i}\right) .
\end{aligned}
$$

The first two terms in (4) simplify to

$$
\frac{2}{r}\left(\left|D^{2} u\right|^{2}-\left|(\nabla u)_{r}\right|^{2}\right)=\frac{2}{r} \sum_{j}\left[\left|\nabla u_{j}\right|^{2}-\left|\partial_{r}\left(u_{j}\right)\right|^{2}\right] .
$$

The remaining terms in (4) are

$$
\begin{aligned}
& 2 u_{i j} u_{i}\left(\frac{1}{r}\right)_{j}+\frac{n-3}{2} u_{j j} u_{i}\left(\frac{1}{r}\right)_{i}-\frac{n-1}{2}\left(\frac{1}{r}\right)_{i} u_{i j j} u \\
& +\left(u_{j} u_{i k}-u_{k} u_{i j}\right)\left(\frac{x_{i} x_{k}}{r^{3}}\right)_{j}-u_{i j} u_{j}\left(\frac{x_{i} x_{k}}{r^{3}}\right)_{k} \\
& \equiv a+b+c+d+e .
\end{aligned}
$$

We evaluate these five terms as follows.

$$
a \approx-u_{i}^{2}\left(\frac{1}{r}\right)_{j j}=\frac{(n-3)}{r^{3}}|\nabla u|^{2} .
$$




$$
\begin{aligned}
b & \approx-\frac{(n-3)}{2}\left(\frac{u_{j}^{2}}{2}\right)_{i}\left(\frac{1}{r}\right)_{i}-\frac{(n-3)}{2} u_{j} u_{i}\left(\frac{1}{r}\right)_{i j} \\
& \approx \frac{(n-3)}{4} u_{j}^{2}\left(\frac{1}{r}\right)_{i i}-\frac{(n-3)}{2} u_{j} u_{i}\left(\frac{1}{r}\right)_{i j} \\
& =-\frac{(n-3)^{2}}{4 r^{3}}|\nabla u|^{2}+\frac{(n-3)}{2 r^{3}}|\nabla u|^{2}-\frac{3(n-3) u_{r}^{2}}{2 r^{3}} \\
& =-\frac{(n-3)(n-5)}{4 r^{3}|\nabla u|^{2}-\frac{3(n-3)}{2 r^{3}} u_{r}^{2}} . \\
c & \approx \frac{(n-1)}{2} u_{i j} u_{j}\left(\frac{1}{r}\right)_{i}+\frac{(n-1)}{2} u_{i j} u\left(\frac{1}{r}\right)_{i j} \\
& \approx-\frac{(n-1)}{4} u_{j}^{2}\left(\frac{1}{r}\right)_{i i}-\frac{n-1}{2} u_{i} u_{j}\left(\frac{1}{r}\right)_{i j}-\frac{n-1}{2} u_{i} u\left(\frac{1}{r}\right)_{i j j} \\
& \approx \frac{(n-1)(n-3)}{4 r^{3}}|\nabla u|^{2}+\frac{n-1}{2 r^{3}}|\nabla u|^{2}-\frac{3(n-1)}{2 r^{3}} u_{r}^{2}+\frac{n-1}{4} u^{2}\left(\frac{1}{r}\right)_{i i j j} \\
& =\frac{(n-1)^{2}}{4 r^{3}}|\nabla u|^{2}-\frac{3(n-1)}{2 r^{3}} u_{r}^{2}+\frac{3(n-1)(n-3)(n-5)}{4 r^{5}} u^{2}
\end{aligned}
$$

provided $n>5$. (The case $n=5$ will be treated later.)

$$
\begin{aligned}
d & =\left(\left(u_{i} u_{j}\right)_{k}-\left(u_{i} u_{k}\right)_{j}\right)\left(\frac{x_{i} x_{k}}{r^{3}}\right)_{j} \\
& \approx-u_{i} u_{j}\left(\frac{x_{i} x_{k}}{r^{3}}\right)_{j k}+u_{i} u_{k}\left(\frac{x_{i} x_{k}}{r^{3}}\right)_{j j} \\
& =-\frac{(n-2)}{r^{3}}|\nabla u|^{2}+\frac{3(n-2)}{r^{3}} u_{r}^{2}+\frac{2}{r^{3}}|\nabla u|^{2}-\frac{3(n-1)}{r^{3}} u_{r}^{2} \\
& =-\frac{(n-4)}{r^{3}}|\nabla u|^{2}-\frac{3}{r^{3}} u_{r}^{2} . \\
e \approx & \frac{1}{2} u_{j}^{2}\left(\frac{x_{i} x_{k}}{r^{3}}\right)_{i k}=\frac{(n-3)(n-2)}{2 r^{3}}|\nabla u|^{2} .
\end{aligned}
$$

Summing the boxed terms leads to the expression

$$
\begin{aligned}
& \left(\Delta^{2} u\right)(B u) \approx \frac{2}{r}\left(\left|D^{2} u\right|^{2}-\left|(\nabla u)_{r}\right|^{2}\right) \\
& \quad+\frac{n-1}{2 r^{3}}\left\{(n-1)|\nabla u|^{2}-6\left|u_{r}\right|^{2}\right\}+(n-1)(n-3)(n-5) \frac{3 u^{2}}{4 r^{5}} .
\end{aligned}
$$

The last term in (6) corresponds to the second-to-last term in (2). We wish to rearrange the other terms in (6) to be manifestly non-negative.

For convenience we introduce the notation

$$
\hat{x}_{i}=\frac{x_{i}}{r}, \quad \partial_{r}=\hat{x}_{i} \partial_{i}, \quad \Omega_{i j}=x_{i} \partial_{j}-x_{j} \partial_{i}
$$


where $\Omega_{i j}$ and $\partial_{r}$ commute. We note that any derivative decomposes into radial and angular parts

$$
\partial_{i}=\hat{x}_{i} \partial_{r}+\frac{\hat{x}_{j}}{r} \Omega_{j i}
$$

that are orthogonal to each other at each point because the matrix $\Omega_{j i}$ is skewsymmetric. The leading expression $\left|D^{2} u\right|^{2}=\sum\left|\partial_{j} \partial_{i} u\right|^{2}$ in (6) is decomposed as follows.

$$
\partial_{i} u=\hat{x}_{i} \partial_{r} u+\frac{\hat{x}_{k}}{r} \Omega_{k i} u
$$

and

$$
\begin{aligned}
\partial_{j} \partial_{i} u= & \hat{x}_{i} \partial_{j} \partial_{r} u+\partial_{j}\left(\hat{x}_{i}\right) \partial_{r} u+\partial_{j}\left\{\frac{\hat{x}_{k}}{r} \Omega_{k i} u\right\} \\
= & \hat{x}_{i}\left\{\hat{x}_{j} \partial_{r}+\frac{\hat{x}_{k}}{r} \Omega_{k j}\right\} \partial_{r} u \\
& +\frac{\delta_{i j}-\hat{x}_{i} \hat{x}_{j}}{r} \partial_{r} u+\partial_{j}\left\{\frac{\hat{x}_{k}}{r} \Omega_{k i} u\right\} \\
= & R_{i j} u+S_{i j} u
\end{aligned}
$$

where

$$
R_{i j} u=\hat{x}_{i} \hat{x}_{j} \partial_{r}^{2} u+\frac{\delta_{i j}-\hat{x}_{i} \hat{x}_{j}}{r} \partial_{r} u
$$

and

$$
S_{i j} u=\frac{\hat{x}_{i} \hat{x}_{k}}{r} \Omega_{k j} \partial_{r} u+\partial_{j}\left\{\frac{\hat{x}_{k}}{r} \Omega_{k i} u\right\}
$$

Squaring, we get

$$
\left|D^{2} u\right|^{2}=\sum_{i j}\left\{\left(R_{i j} u\right)^{2}+2\left(R_{i j} u\right)\left(S_{i j} u\right)+\left(S_{i j} u\right)^{2}\right\} .
$$

Claim 1. The cross terms contribute

$$
\frac{4}{r} \sum\left(R_{i j} u\right)\left(S_{i j} u\right) \approx \frac{2(n-5)}{r^{3}}\left(|\nabla u|^{2}-\left(\partial_{r} u\right)^{2}\right) \geq 0 \text {. }
$$

Indeed, $4 \sum\left(R_{i j} u\right)\left(S_{i j} u\right)$ contains three kinds of terms. The first kind contains the expression $\sum \hat{x}_{j} \hat{x}_{k} \Omega_{k j}$, which vanishes by the skew-symmetry of $\Omega$. The second kind is

$$
\begin{aligned}
4 \hat{x}_{i} \hat{x}_{j}\left(\partial_{r}^{2} u-\frac{1}{r} \partial_{r} u\right) & \cdot \partial_{j}\left[\frac{\hat{x}_{k}}{r} \Omega_{k i} u\right] \\
& =4 \frac{1}{r} \hat{x}_{j}\left(\partial_{r}^{2} u-\frac{1}{r} \partial_{r} u\right) \cdot\left\{\partial_{j}\left[x_{i} \frac{\hat{x}_{k}}{r} \Omega_{k i} u\right]-\delta_{i j} \frac{\hat{x}_{k}}{r} \Omega_{k i} u\right\}=0,
\end{aligned}
$$

in which both terms on the right contain $\sum \hat{x}_{i} \hat{x}_{k} \Omega_{k i}=0$. The third kind is

$$
4 \delta_{i j} \frac{1}{r^{2}} \partial_{r} u \cdot \partial_{j}\left[\frac{\hat{x}_{k}}{r} \Omega_{k i} u\right]=\frac{4}{r^{2}} \partial_{r} u \cdot \partial_{i} A_{i} u \approx-4 \partial_{i}\left(\frac{1}{r^{2}} \partial_{r} u\right) \cdot A_{i} u
$$

where

$$
A_{i}=\frac{\hat{x}_{j}}{r} \Omega_{j i}=\partial_{i}-\hat{x}_{i} \partial_{r}
$$


Observe that

$$
x_{i} A_{i}=0, \quad A_{i}(f(r))=0, \quad A_{i} \partial_{r}=\partial_{r} A_{i}+\frac{1}{r} A_{i} .
$$

Using this notation, we are left with

$$
\begin{aligned}
-4\left(\hat{x}_{i} \partial_{r}+A_{i}\right)\left(\frac{1}{r^{2}} \partial_{r} u\right) \cdot A_{i} u & =-4 A_{i}\left(\frac{1}{r^{2}} \partial_{r} u\right) \cdot A_{i} u \\
& =-\frac{4}{r^{2}} A_{i} \partial_{r} u \cdot A_{i} u=-\frac{4}{r^{2}}\left(\partial_{r} A_{i} u+\frac{1}{r} A_{i} u\right) \cdot A_{i} u \\
& =\left(-\frac{2}{r^{2}} \partial_{r}-\frac{4}{r^{3}}\right) w
\end{aligned}
$$

where

$$
w=\sum_{i}\left(A_{i}\right)^{2}=|\nabla u|^{2}-\left(\partial_{r} u\right)^{2} .
$$

Thus our expression can be written as

$$
\left[-\frac{2 \hat{x}_{i}}{r^{2}} \partial_{i}-\frac{4}{r^{3}}\right] w \approx\left[2 \partial_{i}\left(\frac{\hat{x}_{i}}{r^{2}}\right)-\frac{4}{r^{3}}\right] w=\frac{2(n-5)}{r^{3}} w=\frac{2(n-5)}{r^{3}}\left(|\nabla u|^{2}-\left(\partial_{r} u\right)^{2}\right) .
$$

This proves Claim 1.

Claim 2.

$$
\frac{2}{r}\left|D^{2} u\right|^{2}=\frac{2}{r}\left\{\left(\partial_{r}^{2} u\right)^{2}+\frac{n-1}{r^{2}}\left(\partial_{r} u\right)^{2}+\sum_{i, j}\left(S_{i j} u\right)^{2}\right\}+\frac{2(n-5)}{r^{3}}\left(|\nabla u|^{2}-\left(\partial_{r} u\right)^{2}\right) .
$$

Indeed,

$$
\begin{aligned}
{\left[R_{i j} u\right]^{2} } & =\left(\hat{x}_{i} \hat{x}_{j} \partial_{r}^{2} u+\left(\delta_{i j}-\hat{x}_{i} \hat{x}_{j}\right) \frac{1}{r} \partial_{r} u\right)^{2} \\
& =\hat{x}_{i}^{2} \hat{x}_{j}^{2}\left(\partial_{r}^{2} u\right)^{2}+2 \hat{x}_{i} \hat{x}_{j}\left(\delta_{i j}-\hat{x}_{i} \hat{x}_{j}\right) \partial_{r}^{2} u \cdot \frac{1}{r} \partial_{r} u+\left(\delta_{i j}-\hat{x}_{i} \hat{x}_{j}\right)^{2}\left(\frac{1}{r} \partial_{r} u\right)^{2}
\end{aligned}
$$

When summed, the middle term vanishes while $\sum\left(\delta_{i j}-\hat{x}_{i} \hat{x}_{j}\right)^{2}=n-1$. Thus $\sum\left(R_{i j} u\right)^{2}$ $=\left(\partial_{r}^{2} u\right)^{2}+\frac{n-1}{r^{2}}\left(\partial_{r} u\right)^{2}$. Thus Claim 2 follows from Claim 1 .

Claim 3.

$$
\left|(\nabla u)_{r}\right|^{2} \equiv\left|\partial_{r} \nabla u\right|^{2}=\left(\partial_{r}^{2} u\right)^{2}+\sum_{i}\left(\hat{x}_{j} S_{i j} u\right)^{2} .
$$

Indeed,

$$
\partial_{r} \partial_{i} u=\hat{x}_{j} \partial_{j} \partial_{i} u=\hat{x}_{j}\left(R_{i j} u+S_{i j} u\right) .
$$

This expression must be squared and then summed over $i$. Now

$$
\hat{x}_{j} R_{i j} u=\hat{x}_{j}\left(\hat{x}_{i} \hat{x}_{j} \partial_{r}^{2} u+\frac{\delta_{i j}-\hat{x}_{i} \hat{x}_{j}}{r} \partial_{r} u\right)=\hat{x}_{i} \partial_{r}^{2} u
$$

while

$$
\hat{x}_{j} S_{i j} u=\hat{x}_{j}\left(\hat{x}_{i} A_{i} \partial_{r} u+\partial_{j} A_{i} u\right)=\partial_{r} A_{i} u
$$

Therefore

$$
\hat{x}_{k} R_{i k} u \cdot \hat{x}_{j} S_{i j} u=\hat{x}_{i} \partial_{r}^{2} u \cdot \partial_{r} A_{i} u=\partial_{r}^{2} u \cdot \partial_{r}\left(\hat{x}_{i} A_{i} u\right)=0
$$


because the operators $\partial_{r}$ and $\hat{x}_{i}$ commute and $x_{i} A_{i}=0$. Upon summing and squaring, we end up with Claim 3.

By (6), together with Claims 2 and 3, we conclude that

$$
\begin{aligned}
& \left(\Delta^{2} u\right)(B u) \\
& \approx \frac{2}{r}\left\{\left(\partial_{r}^{2} u\right)^{2}+\frac{n-1}{r^{2}}\left(\partial_{r} u\right)^{2}+\sum_{i j}\left(S_{i j} u\right)^{2}-\left(\partial_{r}^{2} u\right)^{2}-\sum_{i}\left(\sum_{j} \hat{x}_{j} S_{i j} u\right)^{2}\right\} \\
& \quad+\frac{2(n-5)}{r^{3}}\left(|\nabla u|^{2}-\left(\partial_{r} u\right)^{2}\right) \\
& \quad+\frac{n-1}{2 r^{3}}\left\{(n-1)|\nabla u|^{2}-6\left|\partial_{r} u\right|^{2}\right\}+(n-1)(n-3)(n-5) \frac{3 u^{2}}{4 r^{5}} \\
& =(n-1)(n-3) \frac{1}{2 r^{3}}\left(\partial_{r} u\right)^{2}+\frac{n^{2}+2 n-19}{2 r^{3}}\left\{|\nabla u|^{2}-\left(\partial_{r} u\right)^{2}\right\} \\
& \quad+(n-1)(n-3)(n-5) \frac{3 u^{2}}{4 r^{5}}+P
\end{aligned}
$$

where

$$
P=\frac{2}{r}\left\{\sum_{i j}\left(S_{i j} u\right)^{2}-\sum_{i}\left(\sum_{j} \hat{x}_{j} S_{i j} u\right)^{2}\right\}
$$

We note that, by a form of Schwartz' inequality, $P \geq 0$.

In case $n=5$, the calculation of the term $c$ given above must be modified. In fact, in that case

$$
\left(\frac{1}{r}\right)_{i i j j}=\Delta^{2}\left(\frac{1}{r}\right)=16 \pi^{2} \delta(x) .
$$

Thus the last term of $c$ does not vanish but instead equals

$$
-2 u_{i} u\left(\frac{1}{r}\right)_{i j j}=16 \pi^{2}[u(t, 0)]^{2} \geq 0 .
$$

If $n=5$, this non-negative term should be included in $P$. This completes the proof of Theorem 1.

2. Time decay. The following explicit bounds follow from the identity.

Corollary 2. Let $n \geq 5$. Assume $u$ is a solution that is smooth enough and small enough at spatial infinity. Assume

$$
u f(u) \geq 2 F(u) \geq c_{o} u^{2}
$$

for some $c_{o}>0$. Then there is a constant $c$ such that

$$
\begin{gathered}
\int_{0}^{\infty} \int \frac{1}{r}[u f(u)-2 F(u)] d x d t \leq c E \\
\int_{0}^{\infty} \int \frac{1}{r^{3}}|\nabla u|^{2} d x d t \leq c E
\end{gathered}
$$




$$
\begin{gathered}
\int_{0}^{\infty} \int \frac{1}{r^{5}}|u|^{2} d x d t \leq c E \quad \text { provided } n \geq 6 \\
\sup _{x} \int_{0}^{\infty}|u|^{2} d t \leq c E \quad \text { provided } n=5 .
\end{gathered}
$$

Proof. From (1) we get the boundedness of $u$ in $H^{2}\left(\mathbb{R}^{n}\right)$. Therefore the first integral in (2) is bounded by a multiple of $E$. Each of the other terms in (2) is nonnegative. We integrate (2) over a time interval $[0, T]$ and then let $T \rightarrow \infty$. The last term in (2) then gives (10), while the second and third terms in (2) give (11) and the fourth term in (2) gives (12). The estimate (13), special to $n=5$, follows from the term (9) in the proof of Theorem 1 where the origin is shifted to an arbitrary point $x$.

In the typical example $f(u)=u+|u|^{p-1} u$ where $p>1$, we have the integrability in space-time of $r^{-1}|u|^{p+1}$.

We conjecture that the local energy, that is, the norm in $H^{2}(\Omega) \times L^{2}(\Omega)$ for any bounded domain $\Omega \subset \mathbb{R}^{n}$, is integrable and tends to zero as $t \rightarrow \infty$. This conjecture ought to be demonstrable by the same kind of technique as in [M] and [S1].

A solution $u$ of (1) is called a scattering state if there exists a solution $v$ of the linear equation (linearized around the zero solution) such that $\|u(t)-v(t)\| \rightarrow 0$ in the energy norm as $t \rightarrow+\infty$. If $f^{\prime}(0)=1$, the linear equation is

$$
v_{t t}+\Delta^{2} v+v=0
$$

We conjecture that under certain conditions all the states are scattering states. Two main ingredients for proving this conjecture are already known. The first one is a statement that there is some weak decay of solutions of (1). If $n \geq 5$ and the nonlinear term has the repelling sign, this is Corollary 2, especially estimate (10). By the method of $[\mathrm{N}]$ we ought to be able to extend this estimate to a similar one for the lower dimensions $n \leq 4$. The second ingredient is an appropriate decay estimate for the linear equation (14). This too is known, in the form

$$
\|v(t)\|_{L^{q}\left(\mathbb{R}^{n}\right)} \leq C(1+t)^{n / 2 q-n / 4}\|v(0)\|_{X}
$$

where $X=H^{2} \times L^{2}$ is the energy space and $2 \leq q \leq 2+8 /(n-4)$. The upper bound on $q$ can be improved to $\infty$ if $n \leq 6$. Furthermore, there is the space-time estimate

$$
\|v\|_{L^{q}\left(\mathbb{R}^{n+1}\right)} \leq C\|v(0)\|_{X}
$$

for $2+8 / n \leq q \leq 2+12 /(n-4)$. We also note that equation (1) is globally wellposed in energy space if the sign is repelling and $p<1+8 /(n-4)$. Using these ingredients we believe it is possible to prove the scattering conjecture at least for $1+8 / n<p<1+8 /(n-4)$, following the method of [MS] or a variant of it, for smooth solutions and probably also for arbitrary finite-energy solutions. The secondorder analogue is discussed in Chapter 6 of [S2].

\section{REFERENCES}

[B] J. BourgaIn, Global well-posedness of defocusing $3 D$ critical NLS in the radial case, preprint, 1998. 
[Br] P. BRENNER, On scattering and everywhere-defined scattering operators for nonlinear KleinGordon equations, J. Diff. Eq., 56 (1985), pp. 310-344.

[GV1] J. Ginibre And G. Velo, Time decay of finite energy solutions of the nonlinear KleinGordon and Schrödinger equations, Ann. IHP (Phys. Théor.), 43 (1985), pp. 399-442.

[GV2] J. Ginibre and G. Velo, Scattering theory in energy space for a class of nonlinear wave equations, Comm. Math. Phys., 123 (1989), pp. 535-573.

[GV3] J. Ginibre and G. Velo, Scattering theory in the energy space for a class of Hartree equations, preprint, 1998.

[G] M. GRILlakIS, On nonlinear Schrödinger equations, preprint, 1999.

[L1] S. Levandosky, Stability and instability of fourth-order solitary waves, J. Dynam. Diff. Eq., 10 (1998), pp. 151-188.

[L2] S. Levandosky, Decay estimates for fourth-order wave equations, J. Diff. Eq., 143 (1998), pp. 360-413.

[LS] J. E. LiN AND W. A. STRAuSs, Decay and scattering of solutions of a nonlinear Schrödinger equation, J. Funct. Anal., 30 (1978), pp. 245-263.

[M] C. S. Morawetz, Time-decay for the nonlinear Klein-Gordon equation, Proc. Roy. Soc. A, 306 (1968), pp. 291-296.

[MRS] C. S. Morawetz, J. V. Ralston, and W. A. Strauss, Decay of solutions of the wave equation outside trapping obstacles, Comm. Pure Appl. Math., 30 (1977), pp. 447-508.

[MS] C. S. MoraWetz AND W. A. Strauss, Decay and scattering of solutions of a nonlinear relativistic wave equation, Comm. Pure Appl. Math., 25 (1972), pp. 1-31.

[N] K. NAKANISHI, Energy scattering for nonlinear Klein-Gordon and Schrödinger equations in spatial dimensions 1 and 2, J. Funct. Anal., to appear.

[S1] W. A. Strauss, Dispersal of waves vanishing on the boundary of an exterior domain, Comm. Pure Appl. Math., 28 (1975), pp. 265-278.

[S2] W. A. Strauss, Nonlinear Wave Equations, CBMS 73, Amer. Math. Soc., Providence, 1989. 
